\title{
TRANSITION PROBABILITIES FOR SEVERAL INFRARED LINES OF TI I AND Ar I
}

\author{
A. ALONSO-MEDINA \\ Dpto. de Física Atómica, Molecular y Nuclear, Facultad de Ciencias Físicas, Universidad Complutense \\ de Madrid, Avda. Complutense s/n, 28040 Madrid, Spain
}

\begin{abstract}
Relative transition probabilities for 18 infrared (i.r.) lines arising from excited doublets levels of $\mathrm{Tl} I$ and 68 lines belonging to the $\mathrm{Ar}(4 p-3 d),(4 p-5 s)$ and $(4 s-4 p)$ transition array, and coming from the upper levels $3 d[3 / 2]_{1}, 3 d[7 / 2]_{3}, 3 d[5 / 2]_{2}, 3 d^{\prime}[3 / 2]_{2}, 3 d^{\prime}[5 / 2]_{2}$, $3 d^{\prime}[3 / 2]_{1}, 5 s^{\prime}[1 / 2]_{0}, 5 s[3 / 2]_{1}, 5 s[3 / 2]_{2}, 4 p[1 / 2]_{1}, 4 p[5 / 2]_{2}, 4 p[3 / 2]_{1}, 4 p[3 / 2]_{2}, 4 p^{\prime}[3 / 2]_{1}, 4 p^{\prime}[3 / 2]_{2}$ and $4 p^{\prime}[1 / 2]_{1}$ ( $j K$ notation), have been determined from emission line-intensity measurements of an optically-thin light source. Transition probabilities were placed on an absolute scale by using line-strength sum rules. Our experimental results are compared with experimental and theoretical data, where possible, given by other authors.
\end{abstract}

\section{INTRODUCTION}

Relative transition probabilities for $7 s-n p, 6 p-n s$ and $6 p-n d$ i.r. lines of $\mathrm{Tl}$ I have been the subject of numerous theoretical studies using a variety of simple models which have been based on the one-electron central-field approximation, Anderson et al, ${ }^{1}$ Migdalek, ${ }^{2}$ Neuffer and Commins ${ }^{3}$ and Bardsley and Norcross ${ }^{4}$ among others; for these lines there are no published experimental studies, and this situation has prompted the present measurements. In this study I also give transition probabilities for 68 i.r. lines of Ar I which are the subject of few experimental and theoretical studies.

The method used to determine transition probabilities was similar to that employed in previous studies. ${ }^{5-7}$ We measured emission intensities of lines arising from the same upper level to determine relative transition probabilities, whose values become independent of the upper level population when working with optically-thin light sources. ${ }^{8}$ Transition probabilities are obtained on an absolute scale by using line-strength sum-rules, ${ }^{9}$ and the Coulomb approximation for i.r. lines of Ar $I$. The results are compared with the intermediate-coupling calculation of Lilly, ${ }^{10}$ who also used the Coulomb approximation, as well as with previously published experimental results of Wiese, ${ }^{11.12}$ Tanarro $^{5,6}$ and Borge. ${ }^{13}$

Tl I transition probabilities also have been obtained on an absolute scale by using line-strength sum-rules and relativistic semiempirical method which included core-polarization effects, both in the model potential and in the transition matrix element. These experimental results are compared with theoretical calculations performed by other authors. To provide level energies of our calculations, the tables of Moore ${ }^{14}$ have been used.

\section{EXPERIMENTAL SET-UP AND PROCEDURE}

The experimental set-up is similar to that described in a previous publication. ${ }^{7.15}$ The spectral source used was an a.c. $\mathrm{Tl}$ arc lamp operating at $0.9 \mathrm{~A}$, and the light path was $1 \mathrm{~cm}$, the gas used to fill the tube was Ar at a pressure of 1 torr. The wavelengths of the measured transition are in a range from 7000 to $15,000 \AA$; the lines were selected by means of a $1 \mathrm{~m}$ Eagle monochromator, ${ }^{16}$ having a 600 grooves/mm concave holographic grating, blazed at $9000 \AA$ of $2 \AA$ resolution in the 


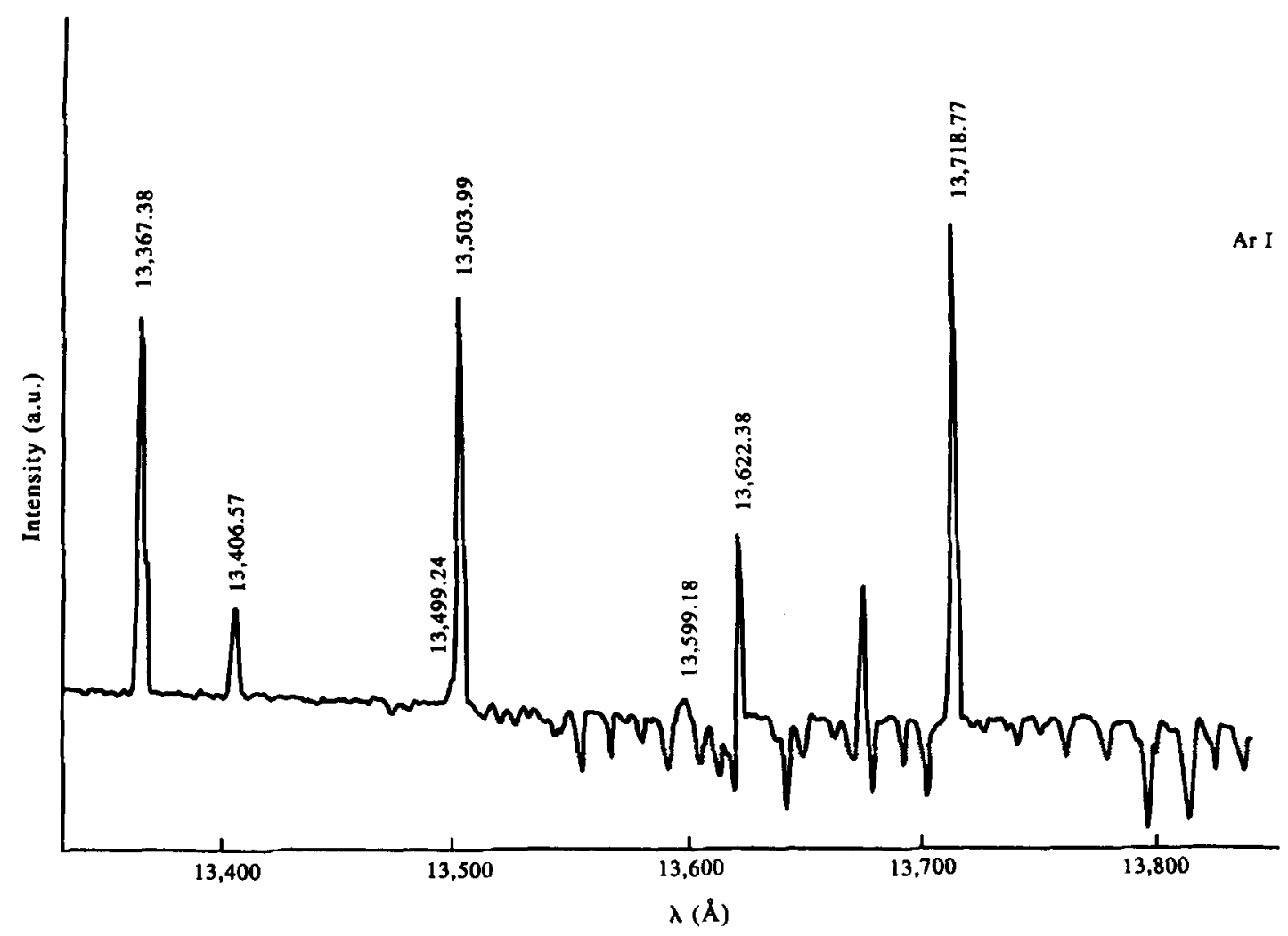

Fig. 1. Spectrum adjacent to the lines of Ar $I$, bands of water vapour which are in the collimator.

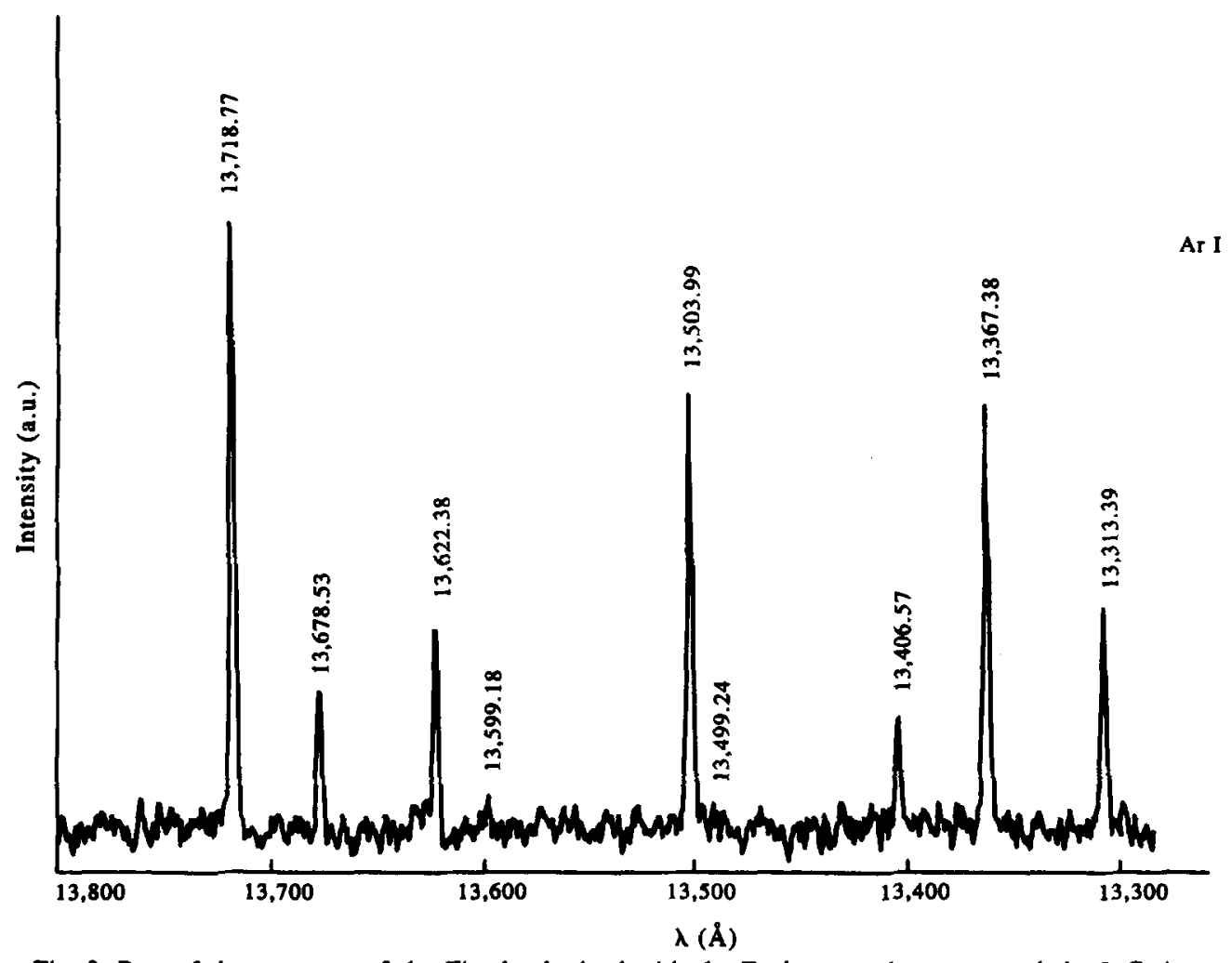

Fig. 2. Part of the spectrum of the Fig. 1, obtained with the Eagle monochromator and the InGaAs detector, in which the i.r. lines of At 1 are shown. 
first order; appropriate cut-off optical filters were used to eliminate second-order radiation. Photons from 6000 to $11,000 \AA$ were detected with an EMI 9898 B (S1 response) photomultiplier cooled with dry ice. Photons of the transition wavelength range, from 8000 to $15,000 \AA$, were detected with an InGaAs PIN photodiode (EPITAXX ETX 3000) operating in the photovoltaic mode; the photosensitive diameter was $3 \mathrm{~mm}$.

To eliminate noise signals, a phase-sensitive detection technique ${ }^{5.17}$ was used; the i.r. light was chopped at a frequency of $450 \mathrm{~Hz}$. The electronic system had consisted of a passband amplifier, an analog-digital converter and an analog phase-sensitive circuit which subtracts the half-periods of noise from the signal plus noise half-periods; the output of this circuit feeds a strip-chart recorder. The wavelength response of the spectrometric system was determined by using a calibrated tungsten-strip lamp; several emission spectra were recorded to obtain relative intensity with statistical incertainties of $5 \%$.

For the i.r. measurements, absorption bands due to water vapour in the ambient air between 13,400 and $14,700 \AA$, were avoided by inserting a drying compound into the monochromator (light path $=2 \mathrm{~m}$ ); this situation can be appreciated in Figs. 1 and 2. The light path from the spectral source to the slit of the monochromator was only $5 \mathrm{~cm}$; corrections due to this effect were negligible within the experimental error. The sensitivity of the detector system alloys measurements with optically-thin sources, avoiding systematic errors due to self-absorption; the T1 I and Ar I lines self-absorption tests were made by doubling the optical path by means of a mirror.

Absolute transition probabilities have also been obtained by combining our experimentally measured branching ratios with line-strength sum rules, viz.

$$
\sum_{J^{\prime}} S_{J J^{\prime}}=[(2 J+1) /(2 l+1)] \cdot l_{\max } \cdot\left|\int P_{n l}(r) \cdot r \cdot P_{n^{\prime \prime}}^{\prime}(r) \mathrm{d} r\right|^{2}
$$

where $S_{j j^{\prime}}$ is the line strength for $|n l, J\rangle \rightarrow\left|n^{\prime} l^{\prime}, J^{\prime}\right\rangle$ transitions and $\int P_{n l}(r) \cdot r \cdot P^{\prime}{ }_{n}^{\prime} \gamma^{\prime}(r) \mathrm{d} r$ is the single-electron radial integral.

Since $\mathrm{Tl}$ is a many-electron system having a relatively simple valence configuration $\left(6 s^{2} n l\right)$, the radial wavefunctions $P_{n l}(r)$ were obtained by numerical integration of the radial Schrödinger equation including three terms in the Hamiltonian (Cowan'):

$$
-\frac{\alpha^{2}}{2}(E-V)^{2}-\frac{\alpha^{2}}{4}\left(\frac{\mathrm{d} V}{\mathrm{~d} r}\right) r \frac{\mathrm{d}}{\mathrm{d} r} r^{-1}+\frac{\alpha^{2}}{2}\left(\frac{\mathrm{d} V}{\mathrm{~d} r}\right) \frac{l s}{r}
$$

\begin{tabular}{|c|c|c|c|c|c|c|}
\hline \multirow{2}{*}{\multicolumn{2}{|c|}{$\begin{array}{l}\text { Transition } \\
\text { levels }\end{array}$}} & \multirow[b]{3}{*}{$\lambda(\AA)$} & \multicolumn{4}{|c|}{ Absolute transition probabilities $\left(10^{6} \mathrm{sec}^{-1}\right)$} \\
\hline & & & \multirow{2}{*}{$\begin{array}{l}\text { Experimental } \\
\text { This work }\end{array}$} & \multicolumn{3}{|c|}{ Theory } \\
\hline Upper & Lower & & & This work & Ref. 1 & Ref. 4 \\
\hline $9 s^{2} S_{12}$ & $\begin{array}{l}7 p^{2} P_{1: 2}^{0} \\
7 p^{2} P_{3: 2}^{0}\end{array}$ & $\begin{array}{l}11,100.3 \\
12,488.7\end{array}$ & $\begin{array}{l}1.15 \pm 0.11 \\
1.44 \pm 0.14\end{array}$ & $\begin{array}{l}1.08 \\
1.49\end{array}$ & $\begin{array}{l}1.18 \\
1.40\end{array}$ & $\begin{array}{l}1.26 \\
1.46\end{array}$ \\
\hline $10 s^{2} S_{12}$ & $\begin{array}{l}7 p^{2} P_{1 / 2}^{0} \\
7 p^{2} P_{3,2}^{0}\end{array}$ & $\begin{array}{l}8976.7 \\
9863.4\end{array}$ & $\begin{array}{l}0.38 \pm 0.04 \\
0.92 \pm 0.09\end{array}$ & $\begin{array}{l}0.54 \\
0.80\end{array}$ & $\begin{array}{l}0.61 \\
0.74\end{array}$ & $\begin{array}{l}0.63 \\
0.72\end{array}$ \\
\hline $11 s^{2} S_{1,2}$ & $\begin{array}{l}7 p^{2} P_{1,2}^{0} \\
7 p^{2} P_{3: 2}^{0}\end{array}$ & $\begin{array}{l}8129.9 \\
8850.5\end{array}$ & $\begin{array}{l}0.34 \pm 0.05 \\
0.42 \pm 0.06\end{array}$ & $\begin{array}{l}0.29 \\
0.45\end{array}$ & $\begin{array}{l}0.34 \\
0.41\end{array}$ & \\
\hline $7 d^{2}=D_{3,2}$ & $\begin{array}{l}7 p^{2} P_{1 / 2}^{0} \\
7 p^{2} P_{3 / 2}^{0}\end{array}$ & $\begin{array}{l}12,732.9 \\
14,593.9\end{array}$ & $\begin{array}{l}7.35 \pm 0.62 \\
1.74 \pm 0.15\end{array}$ & $\begin{array}{l}8.19 \\
1.14\end{array}$ & $\begin{array}{l}5.30 \\
1.31\end{array}$ & $\begin{array}{l}5.10 \\
1.30\end{array}$ \\
\hline $8 d^{2} D_{32}$ & $\begin{array}{l}7 p^{2} P_{1,2}^{0} \\
7 p^{2} P_{3 / 2}^{0}\end{array}$ & $\begin{array}{r}9509.4 \\
10,510.7\end{array}$ & $\begin{array}{l}3.25 \pm 0.23 \\
1.19 \pm 0.09\end{array}$ & $\begin{array}{l}4.11 \\
0.63\end{array}$ & $\begin{array}{l}3.11 \\
0.70\end{array}$ & $\begin{array}{l}3.20 \\
0.67\end{array}$ \\
\hline $9 d^{2} D_{3.2}$ & $\begin{array}{l}7 p^{2} P_{1 / 2}^{0} \\
7 p^{2} \mathbf{P}_{3 / 2}^{0}\end{array}$ & $\begin{array}{l}8373.9 \\
9140.5\end{array}$ & $\begin{array}{l}2.36 \pm 0.16 \\
0.36 \pm 0.02\end{array}$ & $\begin{array}{l}2.36 \\
0.37\end{array}$ & $\begin{array}{l}1.90 \\
0.40\end{array}$ & \\
\hline $10 d^{2} D_{3.2}$ & $\begin{array}{l}7 p^{2} P_{1 / 2}^{0} \\
7 p^{2} P_{3 / 2}^{0}\end{array}$ & $\begin{array}{l}7816.5 \\
8480.3\end{array}$ & $\begin{array}{l}1.68 \pm 0.18 \\
0.20 \pm 0.01\end{array}$ & $\begin{array}{l}1.47 \\
0.23\end{array}$ & $\begin{array}{l}1.21 \\
0.25\end{array}$ & \\
\hline $10 p^{2} P_{3,2}^{0}$ & $\begin{array}{l}6 d^{2} D_{3.2} \\
6 d^{2} D_{5,2}\end{array}$ & $\begin{array}{l}10,072.1 \\
10,156.0\end{array}$ & $\begin{array}{c}0.059 \pm 0.004 \\
0.17 \pm 0.01\end{array}$ & $\begin{array}{l}0.025 \\
0.21\end{array}$ & $\begin{array}{l}0.011 \\
0.16\end{array}$ & \\
\hline $11 p^{2} P_{3: 2}^{0}$ & $\begin{array}{l}6 d^{2} D_{3,2} \\
6 d^{2} D_{5 / 2}\end{array}$ & $\begin{array}{l}9257.4 \\
9328.3\end{array}$ & $\begin{array}{l}0.019 \pm 0.006 \\
0.093 \pm 0.014\end{array}$ & $\begin{array}{l}0.016 \\
0.11\end{array}$ & $\begin{array}{l}0.10 \\
0.095\end{array}$ & \\
\hline
\end{tabular}

Table 1. Transition probubilities for $7 p-n s, 7 p-n a$ and $6 d-n p$ lines of $\mathrm{Tl} \mathrm{I}$. 
where $\alpha$ is the fine structure constant, $E$ is the eigenvalue of the Schrödinger equation, $l$ and $s$ are orbital and spin angular-momentum operators and $V$ is a semiempirical potential given for Green et al, ${ }^{18}$ that includes core polarization effects, ${ }^{19}$ and incorporate the finite sites of nucleus.

The core polarization potential,

$$
V_{\mathrm{p}}(r)=-\frac{1}{2} \alpha_{\mathrm{p}} \frac{r^{2}}{\left(r^{2}+r_{0}^{2}\right)^{3}},
$$

is included in the one-electron Hamiltonian of each valence electron. The static dipole polarizability, $\alpha_{\mathrm{p}}$, of $\mathrm{Tl} \mathrm{I}$ is set to the value (39.33 a.u.) and $r_{0}$ is equated to the mean (2.38 a.u.) of the outermost $(n l j)$ orbital of the unpolarized parent ion, calculated by Fraga et al. ${ }^{20}$ The transition matrix element is corrected for core polarization by replacing the dipole moment operator of the valence electron by

$$
D(r)=r\left[1-\alpha_{\mathrm{p}}\left(r^{2}+r_{0}^{2}\right)^{-3 / 2}\right] .
$$

To incorporate the infinite site of the nucleus, the potential term is given for

$$
V(r)=\frac{Z}{2 R_{0}^{3}} r^{2}-\frac{3}{2} \frac{Z}{R_{0}},
$$

\begin{tabular}{|c|c|c|c|c|c|c|}
\hline \multirow{2}{*}{\multicolumn{2}{|c|}{$\begin{array}{l}\text { Transition } \\
j K \text { notation } \\
\text { levels }\end{array}$}} & \multirow[b]{3}{*}{$\lambda(\AA)$} & \multicolumn{4}{|c|}{ Absolute transition probabilities $\left(10^{6} \mathrm{sec}^{-1}\right)$} \\
\hline & & & \multirow{2}{*}{$\begin{array}{l}\text { Theory } \\
\text { Ref. } 10\end{array}$} & \multicolumn{3}{|c|}{ Experiment } \\
\hline Upper & Lower & & & This work & Ref. 5 & Ref. 11 \\
\hline $3 d[3 / 2]_{1}$ & $\begin{array}{l}4 p[1 / 2]_{1} \\
4 p[5 / 2]_{2} \\
4 p[3 / 2]_{1} \\
4 p[3 / 2]_{2} \\
4 p[1 / 2]_{0}\end{array}$ & $\begin{array}{r}9951.9 \\
11,719.0 \\
12,402.8 \\
12,638.5 \\
14,093.6\end{array}$ & $\begin{array}{l}1.33 \\
9.26 \\
0.12 \\
6.66\end{array}$ & $\begin{array}{c}\leqslant 0.26 \\
1.01 \pm 0.08 \\
10.70 \pm 1.60 \\
\leqslant 0.22 \\
6.46 \pm 1.00\end{array}$ & $\begin{array}{l}\leqslant 0.11 \\
0.8 \pm 0.1 \\
9.7 \pm 0.8 \\
\leqslant 0.13 \\
5.9 \pm 0.7\end{array}$ & $\begin{array}{r}1.07 \\
11.50 \\
4.81\end{array}$ \\
\hline $3 d[7 / 2]_{3}$ & $\begin{array}{l}4 p[5 / 2]_{3} \\
4 p[5 / 2]_{2} \\
4 p[3 / 2]_{2}\end{array}$ & $\begin{array}{l}13,228.5 \\
13,504.0 \\
14,739.1\end{array}$ & $\begin{array}{r}2.72 \\
14.51 \\
0.17\end{array}$ & $\begin{array}{r}2.80 \pm 0.42 \\
13.99 \pm 1.10 \\
0.20 \pm 0.03\end{array}$ & $\begin{array}{l}2.5 \pm 0.3 \\
14.9 \pm 0.8 \\
0.11 \pm 0.20\end{array}$ & $\begin{array}{c}12.2 \\
0.099\end{array}$ \\
\hline $3 d[5 / 2]_{2}$ & $\begin{array}{l}4 p[1 / 2]_{1} \\
4 p[5 / 2]_{3} \\
4 p[5 / 2]_{2} \\
4 p[3 / 2]_{1} \\
4 p[3 / 2]_{2}\end{array}$ & $\begin{array}{l}10,722.2 \\
12,544.4 \\
12,802.7 \\
13,622.4 \\
13,907.4\end{array}$ & $\begin{array}{r}0.35 \\
5.80 \\
11.33 \\
0.22\end{array}$ & $\begin{array}{c}\leqslant 0.13 \\
0.21 \pm 0.04 \\
5.98 \pm 0.59 \\
10.41 \pm 1.02 \\
0.59 \pm 0.09\end{array}$ & $\begin{array}{c}0.14 \\
0.20 \pm 0.07 \\
6.3 \pm 0.6 \\
11.2 \pm 0.8 \\
0.34 \pm 0.08\end{array}$ & $\begin{array}{l}0.14 \\
6.43 \\
8.19\end{array}$ \\
\hline $3 d^{\prime}[3 / 2]_{2}$ & $\begin{array}{l}4 p[5 / 2]_{3} \\
4 \mathrm{p}[5 / 2]_{2} \\
4 p[3 / 2]_{1} \\
4 p[3 / 2]_{2} \\
4 p^{\prime}[3 / 2]_{1} \\
4 p^{\prime}[3 / 2]_{2} \\
4 p^{\prime}[1 / 2]_{\mathrm{t}}\end{array}$ & $\begin{array}{l}10,701.0 \\
10,881.0 \\
11,467.6 \\
11,668.7 \\
13,023.3 \\
13,302.4 \\
13,678.5\end{array}$ & $\begin{array}{c}0.39 \\
0.37 \\
0.52 \\
4.43\end{array}$ & $\begin{array}{c}0.45 \pm 0.07 \\
1.02 \pm 0.15 \\
0.61 \pm 0.06 \\
6.30 \pm 0.94 \\
0.30 \pm 0.04 \\
0.40 \pm 0.04 \\
14.29 \pm 0.0\end{array}$ & $\begin{array}{c}0.40 \pm 0.07 \\
0.9 \pm 0.2 \\
0.58 \pm 0.07 \\
5.6 \pm 0.8 \\
0.25 \pm 0.07 \\
0.30 \pm 0.07 \\
12.0 \pm 0.7\end{array}$ & $\begin{array}{l}0.41 \\
4.23\end{array}$ \\
\hline $3 d^{\prime}[5 / 2]_{2}$ & $\begin{array}{l}4 p^{\prime}[3 / 2]_{1} \\
4 p^{\prime}[3 / 2]_{2} \\
4 p^{\prime}[1 / 2]_{1}\end{array}$ & $\begin{array}{l}13,313.4 \\
13,599.2 \\
13,992.6\end{array}$ & $\begin{array}{r}15.39 \\
0.02\end{array}$ & $\begin{array}{l}15.53 \pm 1.55 \\
2.55 \pm 0.26 \\
\leqslant 0.2\end{array}$ & $\begin{array}{c}15.2 \pm 0.8 \\
2.1 \pm 0.2 \\
\leqslant 0.05\end{array}$ & $\begin{array}{c}14.6 \\
2.54\end{array}$ \\
\hline $3 d^{\prime}[3 / 2]_{1}$ & $\begin{array}{l}4 p[1 / 2]_{1} \\
4 p[5 / 2]_{2} \\
4 p[3 / 2]_{1} \\
4 p[3 / 2]_{2} \\
4 p[1 / 2]_{0} \\
4 p^{\prime}[3 / 2]_{1} \\
4 p^{\prime}[3 / 2]_{2} \\
4 p^{\prime}[1 / 2]_{1} \\
4 p^{\prime}[1 / 2]_{0}\end{array}$ & $\begin{array}{r}8874.8 \\
10,254.0 \\
10,773.4 \\
10,950.7 \\
12,026.6 \\
12,139.8 \\
12,377.2 \\
12,702.3 \\
15,046.5\end{array}$ & $\begin{array}{l}0.07 \\
0.16 \\
0.64 \\
\\
3.34 \\
0.34 \\
5.97 \\
6.25\end{array}$ & $\begin{array}{c}\leqslant 0.18 \\
\leqslant 0.18 \\
0.18 \pm 0.02 \\
0.58 \pm 0.06 \\
0.56 \pm 0.06 \\
6.51 \pm 0.09 \\
0.43 \pm 0.06 \\
8.45 \pm 1.00 \\
6.41 \pm 0.96\end{array}$ & $\begin{array}{c}\leqslant 0.2 \\
\leqslant 0.2 \\
0.22 \pm 0.05 \\
0.57 \pm 0.08 \\
0.55 \pm 0.08 \\
4.5 \pm 0.5 \\
0.23 \pm 0.05 \\
7.2 \pm 0.8 \\
5.1 \pm 0.6\end{array}$ & $\begin{array}{l}0.45 \\
0.47 \\
5.14\end{array}$ \\
\hline
\end{tabular}

where $R_{0}$ is the radius of the nucleus.

Table 2. Transition probabilities for $3 d \rightarrow 4 p$ lines of Ar I 
Table 3. Transition probabilities for $5 s \rightarrow 4 p$ lines of Ar I.

\begin{tabular}{|c|c|c|c|c|c|c|}
\hline \multirow{2}{*}{\multicolumn{2}{|c|}{$\begin{array}{c}\text { Transition } \\
j K \text { notation } \\
\text { levels }\end{array}$}} & \multirow[b]{3}{*}{$\lambda(\AA)$} & \multicolumn{4}{|c|}{ Absolute transition probabilities $\left(10^{6} \mathrm{sec}^{-1}\right)$} \\
\hline & & & \multirow{2}{*}{$\begin{array}{l}\text { Theory } \\
\text { Ref. } 10\end{array}$} & \multicolumn{3}{|c|}{ Experiment } \\
\hline Upper & Lower & & & This work & Ref. 6 & Ref. 11 \\
\hline $5 s[1 / 2]_{0}$ & $\begin{array}{l}4 p[1 / 2]_{1} \\
4 p[3 / 2]_{1} \\
4 p^{\prime}[3 / 2]_{1} \\
4 p^{\prime}[1 / 2]_{1}\end{array}$ & $\begin{array}{r}9291.6 \\
11,393.7 \\
12,933.3 \\
13,573.6\end{array}$ & $\begin{array}{r}2.82 \\
2.58 \\
10.87 \\
5.29\end{array}$ & $\begin{array}{r}4.0 \pm 0.4 \\
3.1 \pm 0.3 \\
11.0 \pm 1.0 \\
5.9 \pm 0.9\end{array}$ & $\begin{array}{r}4.2 \pm 0.6 \\
3.5 \pm 0.5 \\
11.9 \pm 1.5 \\
5.4 \pm 0.6\end{array}$ & $\begin{array}{c}3.66 \\
2.49 \\
10.9\end{array}$ \\
\hline $5 s[3 / 2]_{1}$ & $\begin{array}{l}4 p[1 / 2]_{1} \\
4 p[5 / 2]_{2} \\
4 p[3 / 2]_{1} \\
4 p[3 / 2]_{2} \\
4 p[1 / 2]_{0}\end{array}$ & $\begin{array}{l}10,478.1 \\
12,456.1 \\
13,230.9 \\
13,499.2 \\
15,172.3\end{array}$ & $\begin{array}{l}1.9 \\
8.9 \\
4.6 \\
2.6 \\
1.3\end{array}$ & $\begin{array}{l}2.7 \pm 0.2 \\
6.1 \pm 0.6 \\
2.9 \pm 0.3 \\
\leqslant 0.13 \\
1.0 \pm 0.1\end{array}$ & $\begin{array}{c}3.1 \pm 0.5 \\
10.4 \pm 1.5 \\
\leqslant 2.5 \\
1.5 \pm 0.3\end{array}$ & $\begin{array}{l}1.9 \\
8.9 \\
\\
2.6\end{array}$ \\
\hline $5 s[3 / 2]_{2}$ & $\begin{array}{l}4 p[1 / 2]_{1} \\
4 p[5 / 2]_{3} \\
4 p[5 / 2]_{2} \\
4 p[3.2]_{1} \\
4 p[3 / 2]_{2}\end{array}$ & $\begin{array}{l}10,673.6 \\
12,487.6 \\
12,733.6 \\
13,543.8 \\
13,826.0\end{array}$ & $\begin{array}{r}5.0 \\
10.3 \\
1.9 \\
0.5 \\
3.5\end{array}$ & $\begin{array}{r}5.8 \pm 0.9 \\
13.5 \pm 1.9 \\
0.9 \pm 0.1 \\
0.5 \pm 0.1 \\
1.6 \pm 0.2\end{array}$ & $\begin{array}{c}5.8 \pm 0.8 \\
10.2 \pm 1.5 \\
1.4 \pm 0.3 \\
0.47 \pm 0.01 \\
1.4 \pm 0.3\end{array}$ & $\begin{array}{c}4.9 \\
10.3 \\
1.9 \\
0.48 \\
3.5\end{array}$ \\
\hline
\end{tabular}

\section{RESULTS AND DISCUSSION}

The experimental transition probabilities of lines arising from the $\left(n s^{2} S_{1 / 2} \rightarrow 7 p^{2} P_{1,2,3,2}\right)$, $\left(n d^{2} D_{3 / 2} \rightarrow 7 p^{2} P_{1 / 2.3 / 2}\right)$ and $\left(n p^{2} P_{3 / 2} \rightarrow 6 d^{2} D_{3 / 2.5 / 2}\right)$ transitions of $\mathrm{T} 1 \mathrm{I}$ are shown in Table 1; we include for comparison the theoretical values of Anderson et $\mathrm{al}^{1}$ and Bardsley and Norcross; ${ }^{4}$ there is good agreement.

Transition probabilities obtained in the present work for lines arising from the $\operatorname{Ar} \operatorname{I~} 3 d[3 / 2]_{1}$, $3 d[7 / 2]_{3}, 3 d[5 / 2]_{2}, 3 d^{\prime}[3 / 2]_{2}, 3 d^{\prime}[5 / 2]_{2}, 3 d^{\prime}[3 / 2]_{1}$ levels, in $j K$ notation, are given in Table 2; we include for comparison the experimental values of Tanarro ${ }^{5}$ and Wiese. ${ }^{11}$ Table 3 shows the experimental transition probabilities of lines arising from the $\operatorname{Ar} I 5 s^{\prime}[1 / 2]_{0}, 5 s[3 / 2]_{1}, 5 s[3 / 2]_{2}$ levels; we include for comparison the experimental values of Tanarro ${ }^{6}$ and Wiese. ${ }^{11}$ Table 4 shows the values of the transition probabilities of lines arising from the Ar I $4 p[1 / 2]_{1}, 4 p[5 / 2]_{2}, 4 p[3 / 2]_{1}, 4 p[3 / 2]_{2}, 4 p^{\prime}[3 / 2]_{1}$,

Table 4. Transition probabilities for $4 p \rightarrow 7 s$ lines of Ar I.

\begin{tabular}{|c|c|c|c|c|c|c|}
\hline \multirow{2}{*}{\multicolumn{2}{|c|}{$\begin{array}{l}\text { Transition } \\
j K \text { notation } \\
\text { levels }\end{array}$}} & \multirow[b]{3}{*}{$\lambda(\AA)$} & \multicolumn{4}{|c|}{ Absolute transition probabilities $\left(10^{6} \mathrm{sec}^{-1}\right)$} \\
\hline & & & \multirow{2}{*}{$\begin{array}{l}\text { Theory } \\
\text { Ref. } 10\end{array}$} & \multicolumn{3}{|c|}{ Experiment } \\
\hline Upper & Lower & & & This work & Ref. 13 & Ref. 12 \\
\hline $4 p[1 / 2]_{1}$ & $\begin{array}{l}4 s[3 / 2]_{2} \\
4 s[3 / 2]_{1} \\
4 s^{\prime}[1 / 2]_{0} \\
4 s^{\prime}[1 / 2]_{1}\end{array}$ & $\begin{array}{r}9122.9 \\
9657.8 \\
10,470.0 \\
11,488.1\end{array}$ & $\begin{array}{r}17.42 \\
4.65 \\
0.86 \\
0.17\end{array}$ & $\begin{array}{c}16.01 \pm 2.40 \\
6.07 \pm 0.91 \\
1.06 \pm 0.1 \\
0.21 \pm 0.04\end{array}$ & & $\begin{array}{l}18.9 \pm 0.7 \\
5.43 \pm 0.43 \\
0.98 \pm 0.15 \\
0.19 \pm 0.06\end{array}$ \\
\hline $4 p[5 / 2]_{2}$ & $\begin{array}{l}4 s[3 / 2]_{2} \\
4 s[3 / 2]_{1}\end{array}$ & $\begin{array}{l}8014.8 \\
8424.6\end{array}$ & $\begin{array}{r}9.80 \\
20.80\end{array}$ & $\begin{array}{l}10.47 \pm 1.57 \\
21.44 \pm 3.22\end{array}$ & $\begin{array}{l}10.6 \pm 1.3 \\
22.3 \pm 2.7\end{array}$ & $\begin{array}{l}9.28 \pm 0.24 \\
21.5 \pm 1.07\end{array}$ \\
\hline $4 p[3 / 2]_{1}$ & $\begin{array}{l}4 s[3 / 2]_{2} \\
4 s^{2}[3.2]_{1} \\
4 s^{\prime}[1 / 2]_{0} \\
4 s^{\prime}[1 / 2]_{1}\end{array}$ & $\begin{array}{l}7723.8 \\
8103.7 \\
8667.9 \\
9354.2\end{array}$ & $\begin{array}{r}5.65 \\
25.70 \\
2.60 \\
0.90\end{array}$ & $\begin{array}{l}5.20 \pm 0.78 \\
25.7 \pm 3.8 \\
3.00 \pm 0.45 \\
1.22 \pm 0.18\end{array}$ & $\begin{array}{r}4.9 \pm 0.8 \\
24.3 \pm 2.9 \\
2.9 \pm 0.3 \\
1.3 \pm 0.2\end{array}$ & $\begin{array}{r}5.18 \pm 0.26 \\
25.00 \pm 1.25 \\
2.43 \pm 0.19 \\
1.06 \pm 0.08\end{array}$ \\
\hline $4 p[3 / 2]_{2}$ & $\begin{array}{l}4 s[3 / 2]_{2} \\
4 s[3 / 2]_{1}\end{array}$ & $\begin{array}{l}7635.1 \\
8006.2\end{array}$ & $\begin{array}{r}27.00 \\
4.90\end{array}$ & $\begin{array}{r}27.99 \pm 4.20 \\
8.34 \pm 1.40\end{array}$ & $\begin{array}{r}23.8 \pm 2.8 \\
7.1 \pm 0.8\end{array}$ & $\begin{array}{c}24.5 \pm 1.96 \\
4.9 \pm 0.7\end{array}$ \\
\hline $4 p^{\prime}[3 / 2]_{1}$ & $\begin{array}{l}4 s[3 / 2]_{2} \\
4 s[3 / 2]_{1} \\
4 s^{\prime}[1 / 2]_{0} \\
4 s^{\prime}[1 / 2]_{1}\end{array}$ & $\begin{array}{l}7147.0 \\
7471.2 \\
7948.2 \\
8521.4\end{array}$ & $\begin{array}{r}1.00 \\
0.10 \\
20.40 \\
13.40\end{array}$ & $\begin{array}{c}0.91 \pm 0.13 \\
\leqslant 0.1 \\
18.88 \pm 2.83 \\
14.63 \pm 2.19\end{array}$ & $\begin{array}{l}0.7 \pm 0.1 \\
0.03 \pm 0.01 \\
18.7 \pm 2.1 \\
15.7 \pm 1.7\end{array}$ & $\begin{array}{l}0.62 \pm 0.05 \\
0.02 \pm 0.002 \\
18.6 \pm 1.4 \\
13.9 \pm 1.1\end{array}$ \\
\hline $4 p^{\prime}[3 / 2]_{2}$ & $\begin{array}{l}4 s[3 / 2]_{2} \\
4 s[3 / 2]_{1}\end{array}$ & $\begin{array}{l}7067.2 \\
7383.9\end{array}$ & $\begin{array}{l}4.50 \\
9.20\end{array}$ & $\begin{array}{r}4.53 \pm 0.62 \\
10.52 \pm 1.57\end{array}$ & $\begin{array}{l}4.2 \pm 0.5 \\
9.5 \pm 1.0\end{array}$ & $\begin{array}{c}3.8 \pm 0.3 \\
8.47 \pm 0.68\end{array}$ \\
\hline $4 p^{\prime}[1 / 2]_{1}$ & $\begin{array}{l}4 s[3 / 2]_{2} \\
4 s[3 / 2]_{1} \\
4 s^{\prime}[1.2]_{0} \\
4 s[1 / 2]_{1}\end{array}$ & $\begin{array}{l}6965.4 \\
7272.9 \\
7724.2 \\
8264.5\end{array}$ & $\begin{array}{r}7.40 \\
1.90 \\
12.50 \\
17.20\end{array}$ & $\begin{array}{r}7.00 \pm 1.05 \\
2.10 \pm 0.31 \\
12.10 \pm 1.81 \\
15.80 \pm 1.37\end{array}$ & $\begin{array}{r}6.3 \pm 0.7 \\
1.8 \pm 0.2 \\
11.7 \pm 1.9 \\
16.5 \pm 1.8\end{array}$ & $\begin{array}{r}6.39 \pm 0.32 \\
1.83 \pm 0.09 \\
11.70 \pm 0.58 \\
15.30 \pm 0.76\end{array}$ \\
\hline
\end{tabular}


$4 p^{\prime}[3 / 2]_{2}$ and $4 p^{\prime}[1 / 2]_{1}$ levels; we include the experimental values of Wiese ${ }^{12}$ and Borge $^{13}$ for comparison. The experimental errors of relative transition probabilities given in Tables $1-4$ are the result of statistical uncertainties $(\sim 5 \%)$ and uncertainties in the spectral response determination $(\sim 5 \%)$.

The present results are also compared in Tables 2,3 and 4 with the theoretical values of Lilly, ${ }^{10}$ which were obtained by using the same Coulomb approximation and intermediate-coupling scheme. In general, there is agreement with previously published experimental values and good agreement with the calculations of Lilly.

In summary, in the present measurements, results have been obtained for $\mathrm{Ar} I$ that differ by $\sim 10 \%$ from the mean experimental values and for $\mathrm{Tl} \mathrm{I}$, the results differ by $10-15 \%$ from the mean theoretical values; for these infrared lines there are no published experimental studies.

Acknowledgements - The author appreciates helpful discussions concerning aspects of this work with J. Campos, Director of the Department, and thanks the Unit of Atomic Physics and Laser in C.I.E.M.A.T. (Spain), where part of this work was undertaken.

\section{REFERENCES}

1. E. M. Anderson, E. K. Anderson, and V. F. Trusov, Opt. Spectrosc. (URSS) 22, 471 (1967).

2. J. Migdalek, Can. J. Phys. 54, 118 (1976).

3. D. V. Neuffer and E. D. Commins, Phys. Rev. A16, 844 (1977).

4. J. N. Bardsley and D. W. Norcross, JQSRT 23, 575 (1980).

5. I. Tanarro and J. Campos, JQSRT 36, 345 (1986).

6. I. Tanarro and J. Campos, Can. J. Phys. 63, 1389 (1985).

7. C. Peraza, P. Martín, and J. Campos,. JQSRT 45, 63 (1991).

8. J. M. Bridges and W. L. Wiese, Phys. Rev. A2, 285 (1970).

9. R. D. Cowan, The Theory of Atomic Structure and Spectra, p. 422, Univ. of California Press, Los Angeles, CA (1981).

10. R. A. Lilly, JOSA 66, 245 (1976).

11. W. L. Wiese, J. M. Bridges, R. N. Kornblith, and D. E. Kelleher, JOSA 59, 1206 (1969).

12. W. L. Wiese, J. W. Brault, K. Danzmann, V. Helbig, and M. Kock, Phys. Rev. A39, 2461 (1989).

13. M. J. G. Borge and J. Campos, Physica 119C, 359 (1983).

14. C. E. Moore, Atomic Energy Levels, NMS 467, Vol. 11c, U.S.GPO, Washington, DC (1958).

15. C. Peraza, P. Martín, and J. Campos, JQSRT 46, 455 (1991).

16. P. Martín, M. Ortiz, and J. Campos, An. Fis. B85, 70 (1989).

17. M. L. Meade, J. Phys. E: Sci. Instrum. 15, 395 (1982).

18. A. E. S. Green, D. L. Sellin, and A. S. Zachor. Phys. Rev. 184, 1 (1969).

19. J. Migdalek and W. E. Baylis, J. Phys. B: Atom. Molec. Phys. 12, 2595 (1979).

20. S. Fraga, J. Karwowski, and K. M. S. Saxena, Handbook of Atomic Data, pp. 323, 469, Elsevier, New York, NY (1976). 